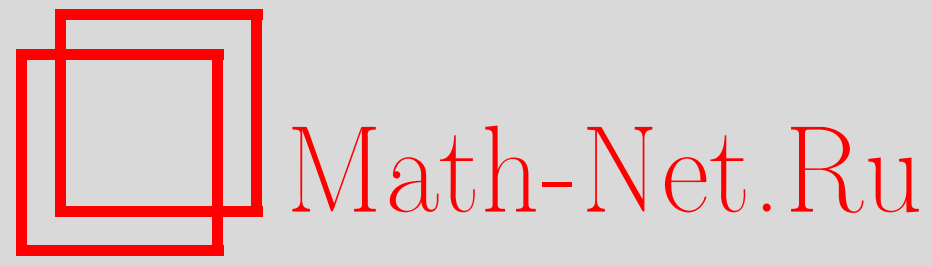

Общероссийский математический портал

Н. В. Крякин, М. Д. Такев, Об интерполяционных константах Уитни, Матем. заметки, 1996, том 59, выпуск 3, 461-463

DOI: https://doi.org/10.4213/mzm1738 
Использование Общероссийского математического портала MathNet.Ru подразумевает, что вы прочитали и согласны с пользовательским соглашением

http://www . mathnet.ru/rus/agreement

Параметры загрузки:

IP: 54.196.121.252

26 апреля 2023 г., 12:38:56

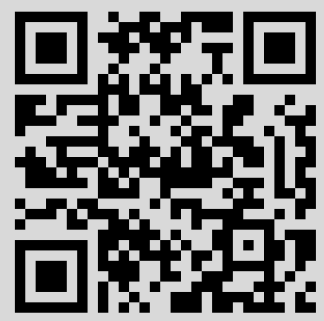




\section{ОБ ИНТЕРПОЛЯЦИОННЫХ КОНСТАНТАХ УИТНИ}

\section{Н. В. Крякин, М. Д. Такев}

1. Для функции определенной на $I=[0,1]$ положим

$$
\Delta_{h}^{n} f(x)=\sum_{j=0}^{n}(-1)^{j}\left(\begin{array}{l}
n \\
j
\end{array}\right) f(x+j h) .
$$

Основным результатом данной заметки является

TEOPEма 1. Пусть $f(x) \in C(I)$ u $Q_{n-1}(f ; x)$ - алгебраический многочлен степени не выше $n-1$ такой, что

$$
f\left(\frac{i}{n-1}\right)=Q_{n-1}\left(f ; \frac{i}{n-1}\right), \quad i=0, \ldots, n-1
$$

Тогда

$$
\sup _{x \in I}\left|f(x)-Q_{n-1}(f ; x)\right| \leqslant W^{\prime}(n) \sup _{y, y+n h \in I}\left|\Delta_{h}^{n} f(y)\right|, \quad c W^{\prime}(n)<5 .
$$

Теорема 1 уточняет оценку интерполяционных констант Уитни $W^{\prime}(n)<38$, полученную в [1].

Отметим, что согласно гипотезе Б. Сендова [2] $W^{\prime}(n)<2$. 
2. Нам понадобится следующее вспомогательное утверждение.

Лемма А [3]. Пусть $f \in C(I), n \in \mathbb{N} u \int_{0}^{1 / n} f(t) d t=0, i=1, \ldots, n$. Тогда для $x \in[1 /(n+1), 1 / n]$

$$
f(i x)=\varphi_{i}(x)-\int_{x}^{1 / n} \sum_{j=1}^{n} \frac{j}{i} \varphi_{j}(y)\left(l_{n, j}\left(\frac{i x}{y}\right)\right)_{x}^{\prime} d y
$$

$2 \partial e$

$$
\begin{gathered}
\varphi_{i}(x)=(-1)^{i}\left(\begin{array}{c}
n \\
i
\end{array}\right)^{-1} \frac{1}{x} \int_{0}^{x} \Delta_{y}^{n} f(i(x-y)) d y, \\
l_{n, j}(t)=\prod_{\substack{k=0 \\
k \neq j}}^{n} \frac{t-k}{j-k}, \quad j=1, \ldots, n .
\end{gathered}
$$

3. Схема доказательства теоремы. Предположив, что

$$
\sup _{y, h}\left|\Delta_{h}^{n} f(y)\right| \leqslant 1
$$

докажем неравенство

$$
\left|f(x)-Q_{n-1}(f ; x)\right|<5 .
$$

Сделаем это косвенньм путем, используя многочлены “интерполяционные в среднем". Они определяются условиями

$$
\int_{0}^{i / n}\left(f(x)-P_{n-1}(f ; x)\right) d x=0, \quad i=1, \ldots, n .
$$

Имеем

$$
\begin{aligned}
\left|f(x)-Q_{n-1}(f ; x)\right| & \leqslant\left|f(x)-P_{n-1}(f ; x)-Q_{n-1}(f ; x)+P_{n-1}(f ; x)\right| \\
& \leqslant\left|f(x)-P_{n-1}(f ; x)\right|+\left|Q_{n-1}\left(f-P_{n-1} ; x\right)\right| .
\end{aligned}
$$

В работе [4] дана оценка первого слагаемого

$$
\left|f(x)-P_{n-1}(f ; x)\right| \leqslant 2 .
$$

Оценка второго слагаемого

$$
\left|Q_{n-1}\left(f-P_{n-1} ; x\right)\right|=\left|\sum_{i=0}^{n-1} \tilde{f}\left(q_{i}\right) l_{n-1, i}(x(n-1))\right|
$$

где

$$
q_{i}=\frac{i}{n-1}, \quad \tilde{f}(x)=f(x)-P_{n-1}(f ; x),
$$

основана на лемме А. Применение этой леммы к $\tilde{f}(x)$ и аккуратный подсчет получающихся сумм приводит к неравенству

$$
\left|\sum_{i=0}^{n-1} \tilde{f}\left(q_{i}\right) l_{n-1, i}(x(n-1))\right|<3 .
$$

Поэтому

$$
\left|f(x)-Q_{n-1}(f ; x)\right|<2+3=5 .
$$

4. Использование многочленов интерполяционных в среднем в качестве промежуточного приближения дает лучший результат при некоторой модификации равномерной точечной интерполяции. Именно, справедлива 
Tеорема 2. Пусть $f(x) \in C(I)$ и $R_{n-1}(f ; x)$ - алгебраический многочлен степени не выше $n-1(n \geqslant 2)$ такой, что

$$
\begin{aligned}
& \text { npu } \quad n=2 k \quad f\left(\frac{i}{n}\right)=R_{n-1}\left(f ; \frac{i}{n}\right), \quad i=0, \ldots, n, \quad i \neq k ; \\
& \text { npu } \quad n=2 k+1 \quad f\left(\frac{i}{n}\right)=R_{n-1}\left(f ; \frac{i}{n}\right), \quad i=0, \ldots, n, \quad i \neq k, k+1 \\
& f\left(\frac{1}{2}\right)=R_{n-1}\left(f ; \frac{1}{2}\right) \text {. }
\end{aligned}
$$

Тогда

$$
\sup _{x \in I}\left|f(x)-R_{n-1}(f ; x)\right| \leqslant 3 \sup _{y, y+n h \in I}\left|\Delta_{h}^{n} f(y)\right| .
$$

Авторы благодарят Б. С. Кашина за внимание к работе.

Одесский политехнический университет

Поступило Sofia University

20.06 .94

\section{СПИСОК ЦИТИРОВАННОЙ ЛИТЕРАТУРЫ}

1. Takev M. On the theorem of Whitney-Sendov // Constructive theory of functions. Sofia, 1992. P. 269-275. 2. Sendov B. // C. R. Acad. Bul. Sci. 1982. V. 35. Р. 431-434. 3. Крякин Ю. В. // Матем. заметки. 1989. Т. 46. № 2. С. 155-157. 4. Крякин Ю.В. Константы Уитни ограничены двойкой // Матем. заметки. 1993. T. 53. № 5. C. 158-160. 